\title{
Combined role of advective pumping and mechanical dispersion on time scales of bed form-induced hyporheic exchange
}

\author{
A. Bottacin-Busolin ${ }^{1}$ and A. Marion ${ }^{1}$ \\ Received 11 November 2009; revised 27 February 2010; accepted 19 April 2010; published 10 August 2010.
}

[1] This study analyzes the effect of advective pumping and pore scale dispersion on bed form-induced hyporheic exchange. Advection and dispersion play a competitive role in the exchange dynamics between the porous medium and the overlying stream: Advective fluxes first lead solutes deep into the bed and then back to the stream water, whereas dispersive fluxes favor the transfer of solutes deep into the bed leading to a permanent mass retention. The combined effect of advective exchange and dispersive fluxes produces complexity in the shape of the tails of the residence time distributions (RTDs), which follow at various stages of the process either a power law or an exponential decay. The seepage velocity induced by the stream gradient and, in case of a moving bed, the celerity of the translating bed forms limit the thickness of the advective hyporheic zone, inducing the RTDs to decrease rapidly at late time. This rapid decay can be preceded by a temporal region where the probability density functions (pdf's) tend to be inversely proportional to the square of time, and is followed by a region dominated by dispersion where the pdf's tend to be inversely proportional to the $3 / 2$ power of time. The process shows distinct temporal ranges identified here by appropriate dimensionless parameters. Because of this complex exchange dynamics, models considering pure advection in the porous medium can significantly underestimate solute transfer at long time scales, whereas purely diffusive models of hyporheic exchange appear inadequate to represent the physical processes at an intermediate stage.

Citation: Bottacin-Busolin, A., and A. Marion (2010), Combined role of advective pumping and mechanical dispersion on time scales of bed form-induced hyporheic exchange, Water Resour. Res., 46, W08518, doi:10.1029/2009WR008892.

\section{Introduction}

[2] The hyporheic zone is the part of a river bed interacting with the streamflow through bidirectional exchanges of mass and momentum. The extension of the hyporheic zone depends both on the characteristics of the surface flow and the sediment bed, which together determine the shape and the length of the flow paths originating from and going back to the stream channel. Hyporheic zones constitute the habitat of many benthic microorganisms [Gibert et al., 1994; Jones and Mulholland, 2000] and are widely recognized to play a crucial role in the evolution of a fluvial ecosystem. In these critical transition zones between the surface and the groundwater a wealth of physical and biogeochemical processes affect the fate of transported substances such as nutrients and contaminants. The capacity of these processes to modify the chemical composition of water depends significantly on the time spent within the sediments. Hyporheic exchange is therefore usually characterized in terms of flux at the stream water-sediment interface and residence time distributions.

[3] A number of models have been proposed to describe the combined dynamics of surface and subsurface solute

\footnotetext{
${ }^{1}$ Department of Hydraulic, Maritime, Environmental and Geotechnical Engineering, University of Padova, Padua, Italy.

Copyright 2010 by the American Geophysical Union. 0043-1397/10/2009WR008892
}

transport in streams and rivers. These models are used in combination with tracer tests in the attempt to extract information about the mechanics of hyporheic exchange by evaluating the effect of transient storage on the concentration of a passive tracer in the surface water. Typically, the mathematical structure of these models consists of a modified one-dimensional advection-dispersion equation with additional retention terms, as in the case of the transient storage model (TSM) presented by Bencala and Walters [1983], and implemented in the widely used OTIS numerical package developed by Runkel [1998]. In the TSM the temporary storage in retention domains is represented as a firstorder mass transfer implying an exponential residence time distribution (RTD). Over the years experimental evidence has shown that this simplified representation of hyporheic exchange is not generally adequate to reproduce the observed breakthrough curves at late times [e.g., Haggerty and Wondzell, 2002; Marion et al., 2003; Zaramella et al., 2003; Gooseff et al., 2007]. To overcome this limitation, a two storage zone extension of the TSM has recently been suggested and applied to a few case studies [Choi et al., 2000; Harvey et al., 2005; Briggs et al., 2009]. General residence time approaches have been proposed based on the theories of multirate mass transfer [Haggerty et al., 2000], continuous time random walk [Boano et al., 2007] and multidomain methods [Marion and Zaramella, 2005; Marion et al., 2008b]. However, even when the observed breakthrough curves are perfectly reproduced, uncertainty can arise concerning the interpretation of the model parameters. The 
uncertainty is primarily a consequence of the superposition of a variety of surface and subsurface retention processes leading to a mathematical indeterminacy of the problem. To solve this issue, a conceptual separation between transient storage in surface dead zones and hyporheic zones can be given in terms of temporal scales by associating the former and the latter to relatively short and long time scales, respectively. On the basis of this conceptual distinction, clever tracer test strategies have been developed for field applications [Briggs et al., 2009].

[4] If enough information is available about the properties of the streamflow and the sediments, general residence time models can also be used as predictive tools, at least in principle. Specific modeling closures can indeed be incorporated to represent retention processes defined on smaller spatial scales than those examined in tracer tests. This is, for example, the idea behind the advective storage path (ASP) model presented by Wörman et al. [2002].

[5] On very short temporal scales the exchange with the bed is dictated by turbulent diffusion across a thin surficial layer of the bed, usually referred to as the Brinkman layer. The interfacial diffusion of momentum was originally investigated by Brinkman [1949] and the resulting interaction between the seepage flow and the free surface turbulent flow above it has subsequently been investigated by a number of authors [Nagaoka and Ohgaki, 1990; Shimizu et al., 1990; Zhou and Mendoza, 1993]. On longer time scales and larger spatial scales, hyporheic flows are induced primarily by the interaction of the streamflow with topographical irregularities of the enclosing boundaries, or by planimetric variations of the flow direction. Topography-driven hyporheic exchange has been particularly studied over the years because of its importance in determining the long-term response of a fluvial system to both natural and anthropic inputs of nutrients and contaminants. Many works have investigated the effects of topographical features such as slope irregularities [Harvey and Bencala, 1993], meanders and channel bends [Boano et al., 2006a; Marion and Zaramella, 2006; Cardenas, 2003, 2008] and alternating bars [Tonina and Buffington, 2007]. Bed form-induced hyporheic exchange in particular has been extensively investigated because bed forms, such as ripples and dunes, are frequently found in rivers and their effect can usually be detected within the typical time scales of tracer tests. Elliott and Brooks [1997a, 1997b] proposed a conceptual model which came to be known as the advective pumping model (APM). In the APM surface and subsurface flow are decoupled and the effect of the former on the latter is represented via a sinusoidal pressure variation imposed on a flat surface representing the top boundary of the bed. Later studies examined how bed form-induced hyporheic exchange is affected by bed form geometry [Marion et al., 2002; Cardenas and Wilson, 2007a], arbitrary surface topography [Wörman et al., 2006, 2007], heterogeneities of the porous medium [Salehin et al., 2004; Marion et al., 2008a; Sawyer and Cardenas, 2009], physicochemical properties of the transported substances [Packman et al., 2000a, 2000b; Packman and Brooks, 2001], unsteady flows [Boano et al., 2006b], and groundwater discharge [Cardenas and Wilson, 2007b; Boano et al., 2008].

[6] In the present work we identify how bed forminduced pumping contributes to determining the RTDs in the sediments by refining Elliott and Brooks's [1997a] approach. A few physical quantities are defined in dimen- sionless form to identify distinct behaviors of the retention process. With this approach we analyze the effect of the stream gradient and the propagation of bed forms on the long-term behavior of the RTDs considering the combined role of advection and pore-scale dispersion in the porous medium. Simulations show that, at late times, the statistical distributions of the residence time in the bed can show both exponential and power law behavior, with different exponents associated to advective pumping and pore scale dispersion. The results provide important insights about the choice of suitable forms of the RTDs to be used in predictive models to identify the signature of hyporheic retention on solute breakthrough curves.

\section{Solute Transport Model in the Porous Medium}

[7] A Cartesian frame of reference is adopted where $x$ is the longitudinal coordinate, oriented along the flow direction, and $y$ is the vertical coordinate, positive upward. Following Elliott and Brooks [1997a], the physical mechanism driving the hyporheic flow in the porous medium is represented by a sinusoidal head distribution imposed on the surface of a semi-infinite flat bed. The wavelength of the head distribution coincides with the bed form length, indicated by $\lambda$, and the amplitude can be linked to the bed form height and the characteristics of the surface flow. The porous medium is considered to be isotropic and homogeneous, with uniform hydraulic conductivity, $K$, and porosity, $\theta$. Additional complexity on the form of the RTD is linked to heterogeneity [Salehin et al., 2004; Marion et al., 2008a; Sawyer and Cardenas, 2009], but the governing behavior at late times described here is general and applies to both homogeneous and heterogeneous systems.

\subsection{Velocity Field}

[8] The interaction between stream water flow and bed forms induces sinusoidal pressure variations over the bed surface [Vittal et al., 1977; Fehlman, 1985]. The pressure is lowest over the crest and separation region downstream of it and rises rapidly to its highest value in the vicinity of the reattachment region near the base of the upslope. These head variations induce flow paths going into the bed where the pressure is higher, and out of the bed where it is lower. The wavelength of the head fluctuations is equal to the bed form wavelength, $\lambda$, and the amplitude depends on the bed form geometry and flow properties. In case of a stationary bed, this pumping mechanism is the main hyporheic exchange process driven by bed forms responsible of the penetration of solutes deep into the bed.

[9] According to Darcy's law, the average pore water (seepage) velocity is given by

$$
\vec{u}=(u, v)=-K \nabla h,
$$

where $u$ and $v$ are the horizontal and vertical components of the Darcy velocity, respectively, and $h(x, y)$ is the hydraulic head in the porous medium. The flow of an incompressible fluid in a homogeneous isotropic bed where Darcy's law holds is governed by the Laplace equation

$$
\nabla^{2} h=\frac{\partial^{2} h}{\partial x^{2}}+\frac{\partial^{2} h}{\partial y^{2}}=0
$$


Equation (2) is solved for a semi-infinite porous medium with the following boundary conditions. On the bed surface, the sinusoidal head variation induced by bed forms is superimposed to the linear decrease due to the energy gradient of the overlying stream flow, whereas the pumping effect vanishes at an infinite depth in the porous medium. In mathematical notation,

$$
\begin{gathered}
h=h_{m} \sin (k x)-S x, \quad y=0, \\
h=-S x, \quad y \rightarrow-\infty,
\end{gathered}
$$

where $k=2 \pi / \lambda$ is the wave number, $h_{m}$ is the semiamplitude of the sinusoidal head over the bed surface, and $S$ is the stream gradient. The semiamplitude $h_{m}$ is related to the characteristics of the overlying stream, such as average velocity and flow depth, and to the bed form geometry (i.e., height and shape). Following Elliott and Brooks [1997a], boundary condition (3) is applied on $y=0$ rather than on the actual bed surface. This simplification well represents hyporheic exchange except at very short time scales because the unevenness of the bed profile induces only minor changes in the length and shape of the flow paths within the porous medium.

[10] Periodic boundary conditions are imposed on the lateral boundaries, that is,

$$
\begin{gathered}
h(x=0, y)=h(x=\lambda, y)+\lambda S, \quad y<0, \\
\frac{\partial h}{\partial x}(x=0, y)=\frac{\partial h}{\partial x}(x=\lambda, y), \quad y<0 .
\end{gathered}
$$

[11] The solution for the hydraulic head is then

$$
h(x, y)=h_{m} \sin (k x) e^{k y}-S x .
$$

Using Darcy's equation (1), the velocity field in the bed is finally recovered:

$$
\begin{aligned}
& u(x, y)=-u_{m} \cos (k x) e^{k y}+u_{s} \\
& v(x, y)=-u_{m} \sin (k x) e^{k y}
\end{aligned}
$$

where $u_{m}=k K h_{m}$ is the semiamplitude of the pumping induced velocity on the bed surface, $y=0$, and $u_{s}=K S$ is the velocity induced by the stream gradient.

[12] The velocity field can be written in nondimensional form using the following normalization for space and time:

$$
x^{*}=k x, \quad y^{*}=k y, \quad t^{*}=\frac{t}{T},
$$

where

$$
T=\frac{\theta}{k^{2} K h_{m}}
$$

is a time scale of hyporheic exchange, and where $\theta$ is the porosity of the bed material. The normalized velocity field can then be given as

$$
\begin{aligned}
& u^{*}=\frac{u}{u_{m}}=-\cos \left(x^{*}\right) e^{y^{*}}+u_{s}^{*} \\
& v^{*}=\frac{v}{u_{m}}=-\sin \left(x^{*}\right) e^{y^{*}}
\end{aligned}
$$

where $u_{s}^{*}=u_{s} / u_{m}=S /\left(k h_{m}\right)$ is the seepage velocity induced by the stream gradient normalized by the characteristic seepage velocity due to pumping. Elliott and Brooks [1997a] performed flume experiments with sand beds covered by bed forms and found values of $u_{s}^{*}$ in the range from 0.04 to 0.11 . They also suggested that this range of values can be considered as typical of field cases where the bed is covered with dunes and form drag dominates the total drag.

[13] The normalized average flux into the bed surface is given by

$$
\begin{aligned}
q^{*} & =-\frac{1}{\lambda u_{m}} \int_{0}^{\lambda / 2} v(x, y=0) d x \\
& =-\frac{1}{2 \pi} \int_{0}^{\pi} v^{*}\left(x^{*}, y^{*}=0\right) d x^{*}=\frac{1}{\pi} .
\end{aligned}
$$

The case of moving bed forms can be handled in a similar way by assuming a frame of reference moving at the speed of bed form propagation, $U_{b}$. In this frame of reference the bed forms appear stationary while the fluid has an additional component of velocity in the streamwise direction equal to $-U_{b}$. Far from the bed surface, deep in the porous medium where the pumping effect is negligible, the pore water appears moving with velocity $u_{s} / \theta-U_{b}$. When $u_{s} / \theta-U_{b}=0$, i.e., if the bed form celerity matches the gradient induced underflow, the velocity field is symmetrical. Typical flow paths for $u_{s} / \theta-U_{b}=0$ are shown in Figure 1. When $u_{s} / \theta-U_{b} \neq 0$, the symmetry disappears and the solutions can be classified in four cases illustrated in Figure 2: $-u_{m}<u_{s}-\theta U_{b}<0$ (Figure 2a), $0<u_{s}-\theta U_{b}<u_{m}$ (Figure 2b), $u_{s}-\theta U_{b}<-u_{m}$ (Figure 2c), and $u_{s}-\theta U_{b}>u_{m}$ (Figure 2d). However, it is important to notice that the interface flux, as well as the vertical component of velocity, are independent of $u_{s} / \theta-$ $U_{b}$, and transport quantities such as penetration depth and residence times are dependent on the magnitude of $u_{s} / \theta-$ $U_{b}$, but are independent of its sign. The analysis can therefore be simplified by considering only the absolute value of the relative underflow velocity, defined in dimensionless form as

$$
U_{u f}^{*}=\left|\frac{u_{s}-\theta U_{b}}{u_{m}}\right|=\left|u_{s}^{*}-U_{b}^{*}\right| .
$$

Although the values of $u_{m}$ and $u_{s}$ are generally not physically independent, their dependence does not affect the discussion presented here and it is not further investigated.

[14] In the case of purely advective flow, for $U_{u f}^{*}=0$ two distinct symmetrical advective cells are visible with flow paths penetrating indefinitely in the porous medium, as shown in Figure 1.

[15] Whenever $U_{u f}^{*} \neq 0$ the hyporheic zone is confined between the streambed interface and a lower boundary defined by a specific streamline. For $U_{u f}^{*}<1$ (Figures 2a and $2 \mathrm{~b}$ ) the flow paths outline two nonsymmetrical cells: a 

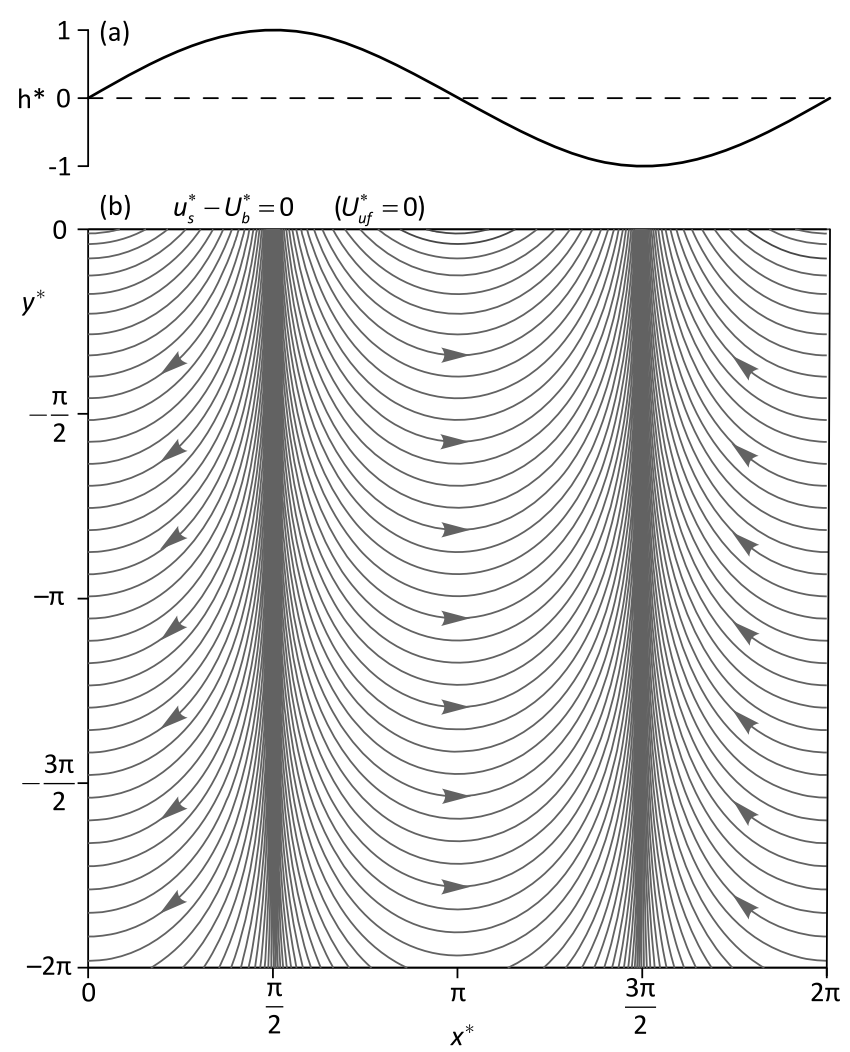

Figure 1. (a) Bed form-induced sinusoidal head distribution and (b) streamlines for $U_{u f}^{*}=0$. The frame of reference moves at the speed of bed form propagation.

larger one where the direction of the average horizontal velocity component is the same as the underflow, and a smaller one where it is opposite. The flow path separating the two advective cells converges in a stagnation point at a finite depth. Situations where $U_{u f}^{*}<1$ are typical of still or slow moving bed forms.

[16] For rapidly moving bed forms $U_{u f}^{*}$ can be higher than 1. For $U_{u f}^{*}>1$ (Figures $2 \mathrm{c}$ and 2d) there is only one advective cell, the streamwise component of the seepage velocity has the same direction of the underflow and there is no stagnation point. The flow path penetrating most deeply in the bed, which outlines the lower boundary of the advective hyporheic zone, reattaches to the bed surface in a point of tangency.

[17] The minimum hyporheic zone depth, for purely advective flows, is the depth of the stagnation point (Figures $2 \mathrm{a}$ and $2 \mathrm{~b}$ ) for $U_{u f}^{*}<1$ and zero for $\mathrm{U}_{u f}^{*}>1$. The maximum hyporheic depth is given by the deepest point crossed by a streamline going into and out of the bed. This is located at $x^{*}=\pi$ when the direction of the relative underflow velocity is positive, and $x^{*}=0,2 \pi$ when it is negative. The average penetration depth is calculated as the total area of the hyporheic zone divided by $\lambda$. Figure 3 shows a plot of the minimum, maximum and average penetration depth of the hyporheic zone as a function of the relative underflow velocity $U_{u f}^{*}$ in both semi-log scale ( $U_{u f}^{*}$ in log-scale) (Figure 3a) and log-log scale (Figure 3b). The penetration depth decreases linearly with $\log \left(U_{u f}^{*}\right)$ for $U_{u f}^{*}<1$ and is inversely proportional to $U_{u f}^{*}$ for $U_{u f}^{*}>1$. An expression that well approximates the average depth of the hyporheic zone as a function of $U_{u f}^{*}$ is the following:

$$
d^{*}= \begin{cases}\frac{e}{\pi}-\log \left(U_{u f}^{*}\right), & U_{u f}^{*}<1 \\ \frac{e}{\pi}\left(U_{u f}^{*}\right)^{-1}, & U_{u f}^{*} \geq 1\end{cases}
$$

where $e$ is the natural base of logarithms.

\subsection{Advection-Dispersion Model}

[18] The transport equation for a nonreactive solute in a porous streambed can be written as [Zheng and Bennett, 1995]

$$
\theta \frac{\partial C}{\partial t}+\frac{\partial}{\partial x_{i}}\left[u_{i} C-\theta D_{i j} \frac{\partial C}{\partial x_{j}}\right]=0
$$

where $C$ is the solute concentration, $u_{i}$ are the components of the seepage (Darcy) velocities, $D_{i j}$ are the components of the hydrodynamic dispersion tensor given by

$$
\theta D_{i j}=\alpha_{T}|\vec{u}| \delta_{i j}+\left(\alpha_{L}-\alpha_{T}\right) \frac{u_{i} u_{j}}{|\vec{u}|} .
$$

[19] In this study the description of the flow field is limited to two dimensions, and hence $i, j=1,2$. In equation (16), $|\vec{u}|$ is the magnitude of the seepage velocity, $\delta_{i j}$ is Kronecker's delta, and $\alpha_{L}$ and $\alpha_{T}$ are the longitudinal and transverse dispersivities, respectively. In most applications, the longitudinal dispersivity can be assumed to be approximately equal to the mean particle size [Qian et al., 2008].

[20] Introducing the normalized quantities

$$
C^{*}=\frac{C}{C_{0}}, \quad u_{i}^{*}=\frac{u_{i}}{u_{m}},
$$

where $C_{0}$ is a reference concentration, and

$$
D_{i j}^{*}=\frac{\theta D_{i j}}{K h_{m}}=\frac{k \theta D_{i j}}{u_{m}}=\alpha_{T}^{*}\left|\vec{u}^{*}\right| \delta_{i j}+\left(\alpha_{L}^{*}-\alpha_{T}^{*}\right) \frac{u_{i}^{*} u_{j}^{*}}{\left|\vec{u}^{*}\right|},
$$

where $\alpha_{L}^{*}=k \alpha_{L}$ and $\alpha_{T}^{*}=k \alpha_{T}$ are the dimensionless dispersivities, equation (15) can be written in dimensionless form:

$$
\frac{\partial C^{*}}{\partial t^{*}}+\frac{\partial}{\partial x_{i}^{*}}\left[u_{i}^{*} C^{*}-D_{i j}^{*} \frac{\partial C^{*}}{\partial x_{j}^{*}}\right]=0 .
$$

[21] When adopting a frame of reference moving at the speed of bed form propagation, equation (19) remains unaltered and $x_{i}$ and $u_{i}$ are respectively the spatial coordinates and the velocity components relative to the moving frame of reference. However, the velocity components defining the dispersion tensor in (18) are those relative to the fixed frame of reference and not the relative ones.

[22] Equation (19) is solved using a modified code from the CLAWPACK software library [LeVeque, 1997]. The 


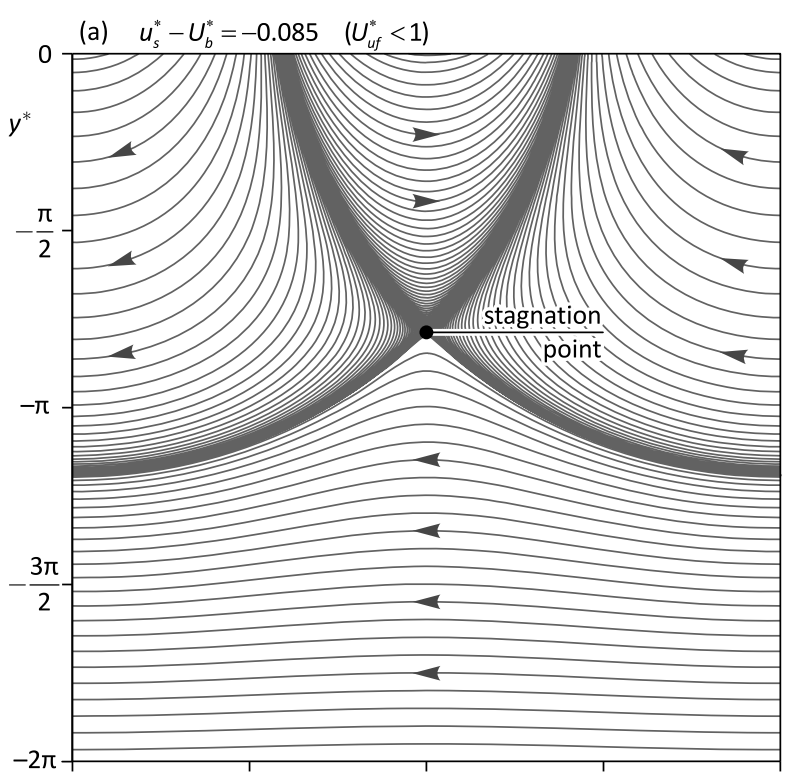

(c) $u_{s}^{*}-U_{b}^{*}=-2.0 \quad\left(U_{u f}^{*}>1\right)$

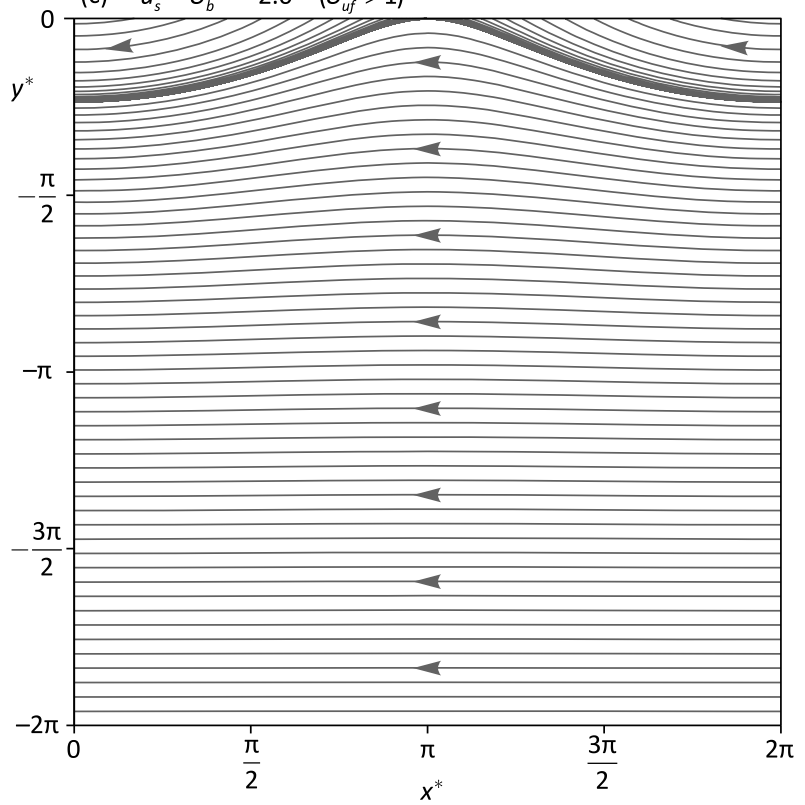

(b) $\quad u_{s}^{*}-U_{b}^{*}=0.085\left(U_{u f}^{*}<1\right)$

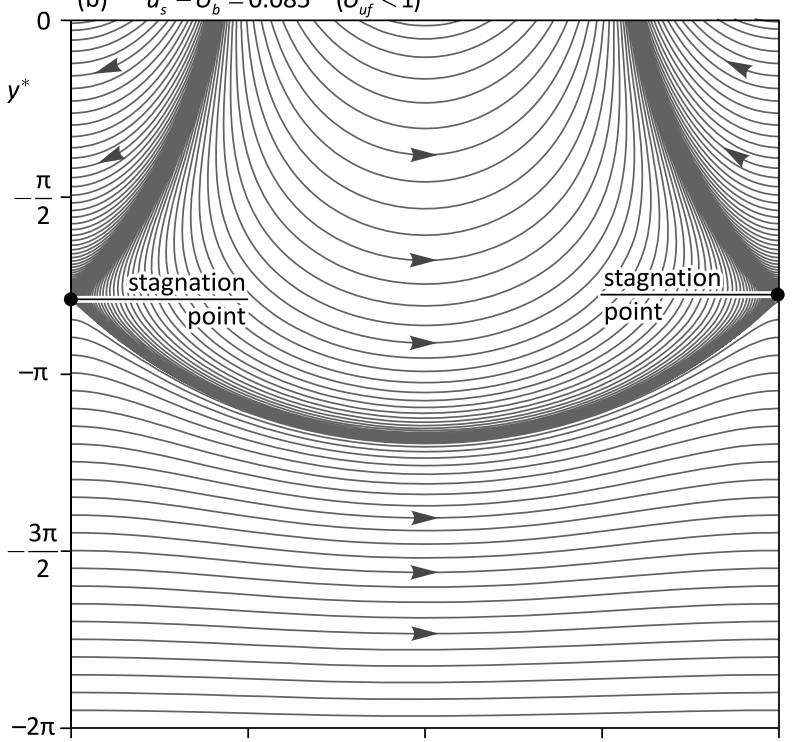

(d) $u_{s}^{*}-U_{b}^{*}=2.0 \quad\left(U_{u f}^{*}>1\right)$

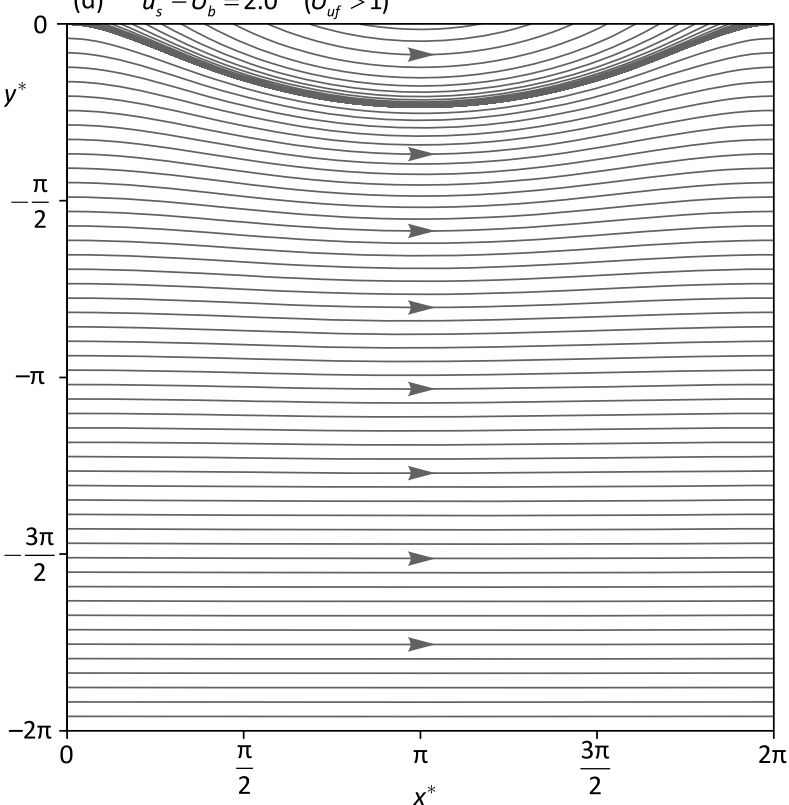

Figure 2. Streamlines of bed form-induced hyporheic exchange for (a) $u_{s}^{*}-U_{b}^{*}=-0.085$, (b) $u_{s}^{*}-U_{b}^{*}=$ 0.085 , (c) $u_{s}^{*}-U_{b}^{*}=-2.0$, and (d) $u_{s}^{*}-U_{b}^{*}=2.0$. The frame of reference moves at the speed of bed form propagation. For $U_{u f}^{*}<1$, two advective cells are visible: a larger cell where the average flow direction is the same as the underflow and a smaller one where it is opposite. The latter shrinks as $U_{u f}^{*}$ increases and disappears for $U_{u f}^{*}>1$.

equation is discretized using a finite volume method which is essentially second-order accurate for smooth concentration distributions and which can also handle step gradients in $C^{*}$ (even discontinuities if $\left.D_{i j}^{*} \equiv 0\right)$. This is accomplished via a fractional step method solving the advection portion of the equation with a high resolution shock-capturing method and the diffusion equation with an implicit finite volume discretization. The details of the algorithm are described in a work by Calhoun and LeVeque [2000]. In solving the equation we tested for grid independence and also checked the solutions for independence on the depth of the lower domain boundary where a no-flux condition was applied, so that the solutions can be regarded as relevant to a semiinfinite bed.

\section{Residence Time Distributions}

[23] Residence time modeling has become increasingly important in the recent years. A number of contributions have recently appeared using general residence time approaches to describe the combined surface and subsurface solute transport in rivers [Haggerty et al., 2000; Marion and Zaramella, 

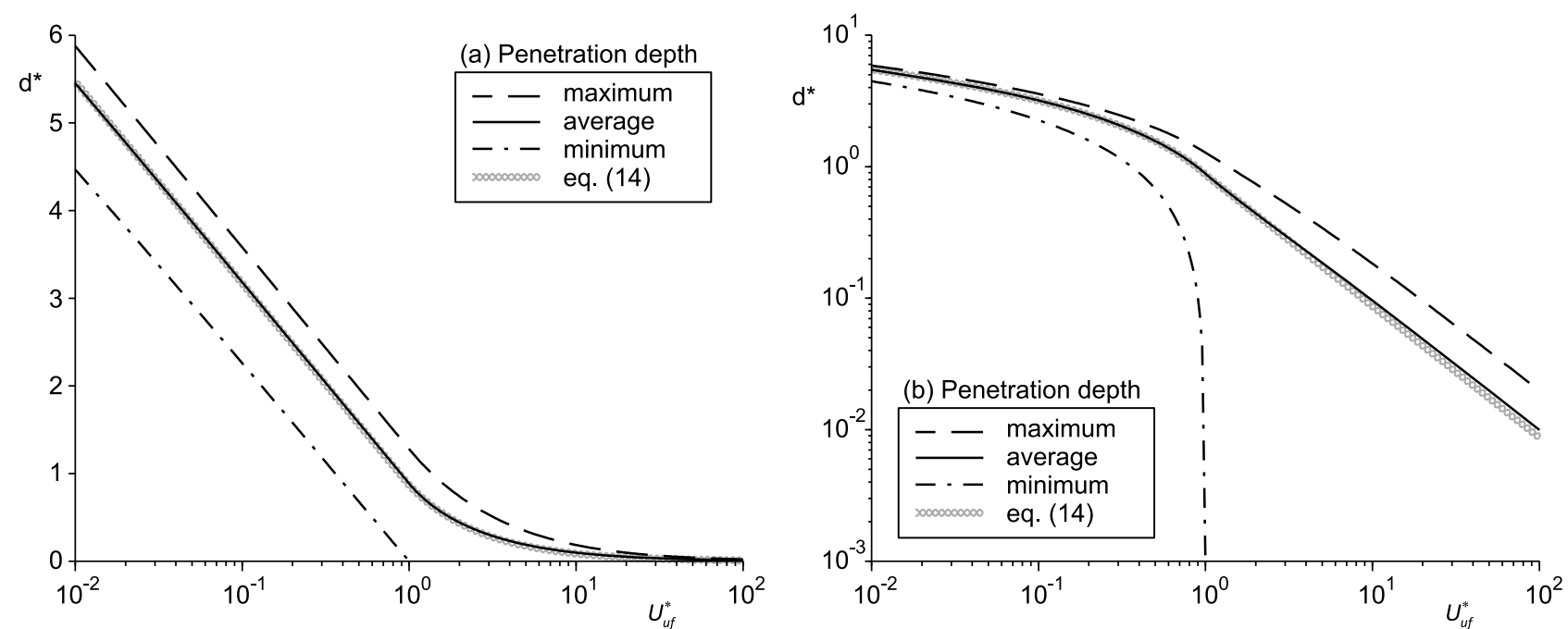

Figure 3. Penetration depth of bed form-induced hyporheic exchange. The dashed curve represents the maximum penetration depth due to advective pumping, i.e., the distance from the bed surface of the deepest point crossed by streamlines going into and out of the bed. The dash-dotted curve represents the depth of the stagnation point, vanishing for $U_{u f}^{*}>1$. The solid curve represents the average penetration depth, i.e., the average thickness of the hyporheic zone.

2005; Boano et al., 2007; Marion et al., 2008b]. These models employ residence time distributions as transfer functions to account for the complex transport dynamics in the storage zones, in particular in the hyporheic zones. The effect of the transient storage on the solute concentration in the surface stream can thus be given in terms of the flux entering the retention domains and the distribution of the residence time within them. Following Elliott and Brooks [1997a], in this work we use residence time function $R$, i.e., the complementary cumulative residence time distribution. For a mass pulse entering the porous medium at time $t_{0}=0$, the function $R(t)$ returns the fraction of solute still in the bed at time $t$. The corresponding probability density function (pdf) of the residence time is given by $r(t)=-d R / d t$.

\subsection{Pure Advection}

[24] Elliott and Brooks [1997a] analyzed the solute exchange induced by pressure variation on the surface considering the effect of pure advection in the porous medium. In this case the residence time distribution can be found using a numerical particle tracking technique applied to the known flow field in the porous medium. Elliott and Brooks provided an analytical solution for the residence time distribution in the idealized case of a bed without stream gradient. The solution was given in implicit form as

$$
t^{*}=\frac{2 \cos ^{-1} R_{0}}{R_{0}}
$$

[25] The residence time function $R_{0}$ solution of equation (20) is a long tail distribution which for $t \rightarrow \infty$ follows a power law with $R \propto t^{-1}$. This means that the corresponding proba- bility density function, $r(t)$, takes the asymptotic form $r \propto t^{-2}$. A closed form approximation of the solution of equation (20) is proposed here as follows:

$$
R_{0}\left(t^{*}\right)=1-\frac{\pi}{4}\left(\frac{1-e^{-\beta t^{*}}}{\beta}-\frac{2}{1+2 / t^{*}}\right)
$$

where

$$
\beta=\frac{\pi}{2(\pi-2)}
$$

[26] The function (21) was found by direct comparison of different functional forms approximating the numerical solutions of equation (20) and represents an alternative of the probability density function given by Marion and Zaramella [2005]. This new form is easier to handle because it has a closed form integral and has a simpler Laplace transform (which is often useful for semi-analytical solution of stream transport models).

[27] In the case of a gradient induced underflow the RTD no longer follows a power law indefinitely, because the flow paths are confined in a zone of finite thickness. When $U_{u f}^{*}<1$, the power law of the tail is visible only up to a given time scale. The presence of a stagnation point in the flow field (Figures $2 \mathrm{a}$ and $2 \mathrm{~b}$ ) implies that there is no finite maximum residence time, but at longer times the $R$ function rapidly decays to zero following an exponential law. In order to give an analytical form to $R\left(t^{*}\right)$ it must be noted that $R\left(t^{*}\right)$ overlaps with $R_{0}\left(t^{*}\right)$ (equation $(21)$ ) at initial times and that it follows an exponential decay as $t^{*} \rightarrow \infty$. Inspection of the form of $R\left(t^{*}\right)$ leads to an analytical approximation in which two time scales are specifically introduced as a 


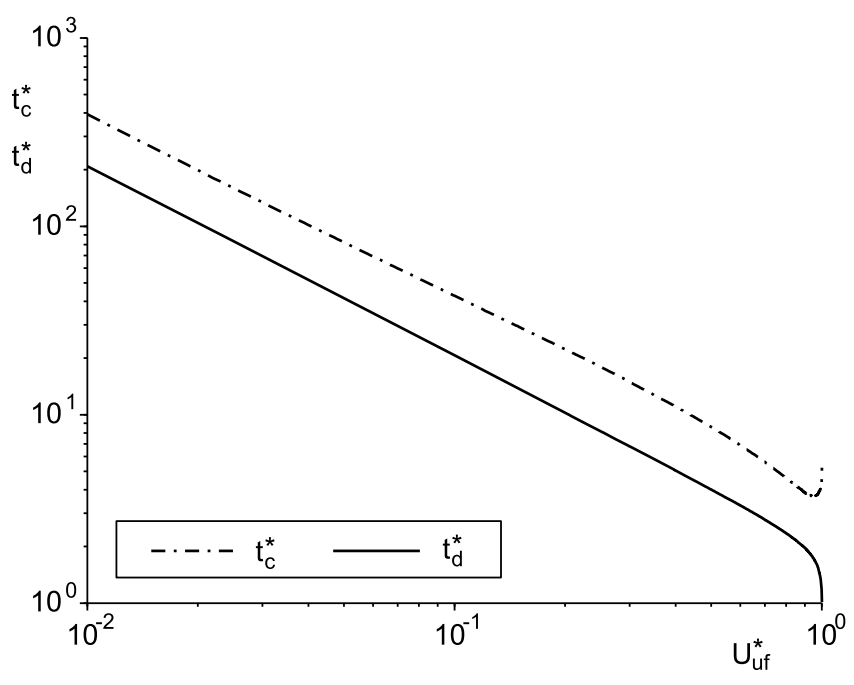

Figure 4. Time scales of the residence time distributions for $U_{u f}^{*}<1$ in case of pure advective transport in the porous medium. Here $t_{c}^{*}$ represents the time scale at which the residence time function $R$ departs from the ideal solution for $U_{u f}^{*}=0$, namely, $R_{0}$, and begins to decrease exponentially with decay rate $t_{d}^{*-1}\left(R \sim e^{-\mathrm{t}^{*} / t_{d}^{*}}\right)$.

function of $U_{u f}^{*}$. The function $R$ is shown to be approximated extremely well by the expression

$$
\begin{aligned}
R\left(t^{*} ; U_{u f}^{*}\right)= & R\left(t^{*} ; U_{u f}^{*}, t_{c}^{*}\left(U_{u f}^{*}\right), t_{d}^{*}\left(U_{u f}^{*}\right)\right) \\
= & \frac{R_{0}\left(t^{*}\right) \exp \left[-\left(t^{*} / t_{c}^{*}\right)^{\left.1+U_{u f}^{*}\right]}+R_{0}\left(t_{c}^{*}\right)\right.}{1+R_{0}\left(t_{c}^{*}\right)} \\
& \cdot \frac{1+e^{t_{c}^{*} / t_{d}^{*}}}{e^{t^{*} / t_{d}^{*}}+e^{t_{c}^{*}} / t_{d}^{*}}, \quad \text { for } U_{u f}^{*}<1,
\end{aligned}
$$

where $t_{c}^{*}$ and $t_{d}^{*}$ are two time scales linked to the velocity $U_{u f}^{*}$. They can be determined by fitting (23) to the residence time functions calculated by numerical particle tracking. The values of $t_{c}^{*}$ and $t_{d}^{*}$ resulting from the least squares fitting of $R$ for $U_{u f}^{*}$ in the range between 0.01 and 1 are plotted in Figure 4. A comparison between the residence time function numerically derived and the approximating function (23) is shown in Figure 5 for a few values of the velocity $U_{u f}^{*}$ logarithmically spaced in the range between 0.01 and 0.95 . As shown in Figure $4, t_{c}^{*}$ and $t_{d}^{*}$ appear to follow a power law except in a small range of values of $U_{u f}^{*}$ very close to 1 , where the approximation error associated to (23) rapidly increases. An approximation for $t_{c}^{*}$ and $t_{d}^{*}$ is given by

$$
t_{c}^{*}=2 t_{d}^{*}=\frac{4}{U_{u f}^{*}} .
$$

When the function (23) is used in combination with (24), the mean relative error of $\log (R)$ for $R$ values in the range $10^{-7}<$ $R<1$ is less than $3 \%$ for $U_{u f}^{*}<0.66$ and less than $5 \%$ for $U_{u f}^{*}<0.75$.

[28] To understand the meaning of the time scales $t_{c}^{*}$ and $t_{d}^{*}$, we observe that at early times, i.e., $t^{*} \ll t_{d}^{*} \ll t_{c}^{*}, R\left(t^{*}\right) \rightarrow$ $R_{0}\left(t^{*}\right)$. Another property of (23) is that, for $t^{*}>t_{c}^{*}>t_{d}^{*}$, $R\left(t^{*}\right) \sim e^{-t^{*} / t^{*}}$, where the tilde symbol means "asymptotically proportional to." In a semi-log graph the tail of $R$ follows a straight line and the slope is $-t_{d}^{*-1}$. The time $t_{c}^{*}$ can be considered as the time scale at which $R\left(t^{*}\right)$ diverges from the idealized behavior given by $R_{0}\left(t^{*}\right)$ and begins to decrease exponentially with decay rate $t_{d}^{*-1}$. Since $R_{0}$ exhibits a power law behavior for about $t^{*}>10$, with $R_{0} \sim t^{*-1}, t_{c}^{*}$ can be seen as the time scale for transition between the power law and the exponential decay of $R$, provided that $t_{c}^{*} \gg 10$ (i.e., $U_{u f}^{*} \ll 0.4$ ). The in-stream breakthrough curve would then be expected to decay to Gaussian for $t^{*}>t_{c}^{*}$. This can be regarded as an expanded version of Fischer et al.'s [1979] criteria for applicability of 1-D Taylor dispersion in rivers.

[29] When the velocity of the underflow is higher than maximum pumping velocity $\left(U_{u f}^{*}>1\right)$, the flow paths are confined in a single advective cell, and a stagnation point is no longer present. From a residence time perspective, this implies the existence of a finite maximum residence time, $t_{\max }^{*}$. For $U_{u f}^{*} \gg 1, t_{\max }^{*}$ is linked to $U_{u f}^{*}$ by the approximation

$$
t_{\max } *=2 \pi\left(U_{u f}^{*-1}+U_{u f}^{*-4}\right),
$$

and the residence time is beta-distributed in the interval [0, $\left.t^{*}{ }_{\max }\right]$. However, for $U_{u f}^{*} \gg 1$ the advective zone is so thin that the effect of the real shape of the bed forms, both in terms of height and aspect ratio, becomes significant, and the sinusoidal model no longer provides an adequate description of the interfacial exchange dynamics. Nevertheless, the sinusoidal head model can still be used to assess, at least qualitatively, the relative importance of advective pumping and mechanical dispersion.

\subsection{Effect of Pore-Scale Dispersion}

[30] The residence time distributions in the porous medium are derived by solving the advection-dispersion equation (19) for a step injection of a constant concentration $C_{0}^{*}=1$ at the downwelling boundary, $0 \leq x^{*} \leq \pi$,

$$
\begin{gathered}
C^{*}=0, \quad t^{*}<0, \\
C^{*}=C_{0}^{*}=1, \quad t^{*} \geq 0 .
\end{gathered}
$$

A convective boundary condition (i.e., Neumann condition with zero dispersive flux) is imposed on the upwelling boundary, $\pi \leq x^{*} \leq 2 \pi$.

[31] The residence time function is then calculated as

$$
R\left(t^{*}\right)=1-\frac{1}{2 \pi q^{*} C_{0}^{*}} \int_{\pi}^{2 \pi} v^{*}\left(x^{*}, y^{*}=0\right) C^{*}\left(x^{*}, y^{*}=0, t^{*}\right) d x^{*}
$$

where $2 \pi q^{*}=2$ is the flow rate per unit width into the bed at the downwelling boundary.

[32] Figure 6 presents the simulated residence time functions resulting from the advection-dispersion model compared to the corresponding functions in case of pure advective transport in the porous medium. The curves in Figure 6 were obtained for $u^{*}{ }_{s}=0.1$ considering values of $U_{u f}^{*}$ in the range $0-5$ and three different values of the dispersivities, namely, $\alpha_{L}^{*}=0.006$ (Figure 6a), $\alpha_{L}^{*}=0.06$ (Figure $6 \mathrm{~b}$ ), and $\alpha_{L}^{*}=0.6$ (Figure $6 \mathrm{c}$ ), thus covering a broad range of situations. In the simulations the ratio $\alpha_{T} / \alpha_{L}$ was assumed to be constant and equal to $1 / 3$ as suggested in other studies [Benekos, 2005; Qian et al., 2008; Zheng and 

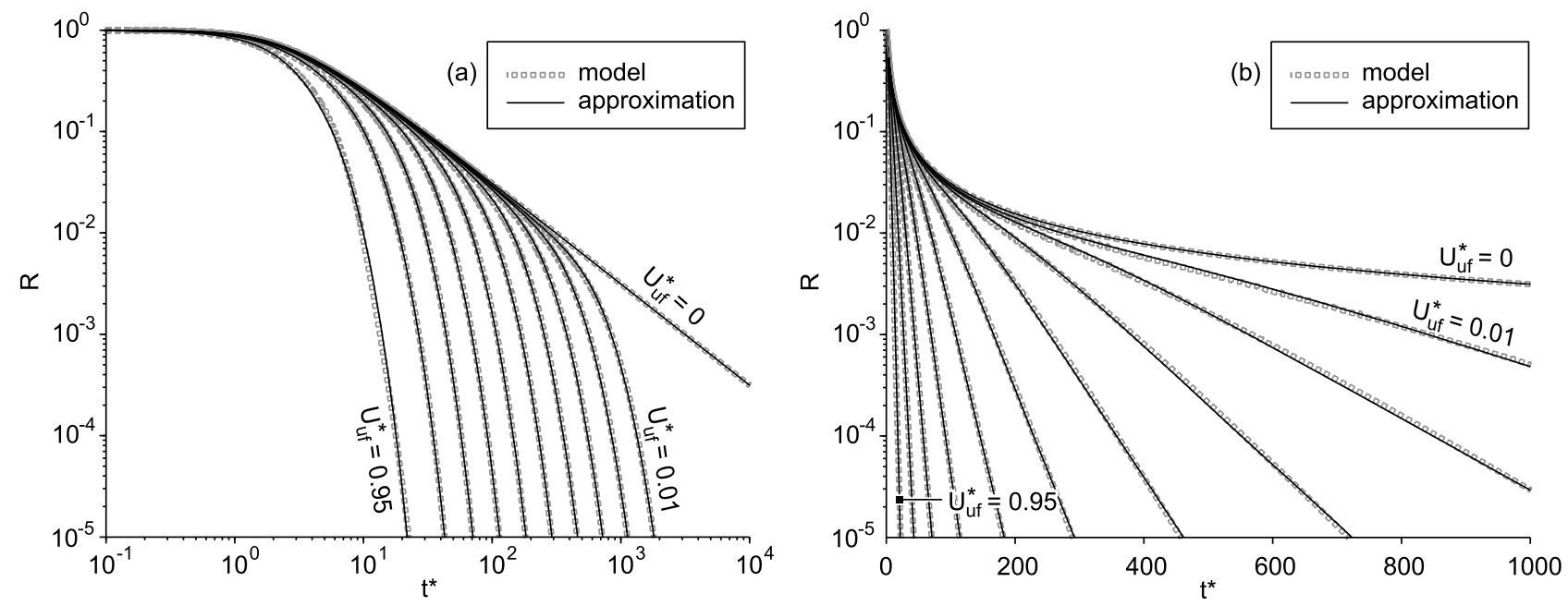

Figure 5. Complementary cumulative distributions of the residence time within the bed for purely advective transport plotted in (a) $\log$ linear scale and (b) log-log scale. The gray curves represent the distributions obtained by numerical particle tracking and account solely for the advective transport in the porous medium. The solid black curves represent analytical approximations given by equation (23). The residence time distributions (RTDs) display a power law behavior where $R \sim t^{-1}$ up to a certain time scale after which the probability decays exponentially with time.

Bennett, 1995]. Note that each distribution must be characterized both in terms of $U_{u f}^{*}$ and $u_{s}^{*}$ because even when adopting a moving frame of reference the dispersion tensor still depends on the actual seepage velocity, and not the relative one. With dispersive transport, the residence time distribution depends on additional parameters compared to the purely advective case. The RTD depends on three parameters (at least): the relative underflow velocity $U_{u f}^{*}$, the seepage velocity induced by the stream gradient $u_{s}^{*}$ and the dispersivity $\alpha_{L}^{*}$, the ratio $\alpha_{T} / \alpha_{L}$ being taken as fixed.

[33] Pore-scale dispersion induces longer tails in the residence time distributions (Figure 6) by producing a net downstream directed flux at the interface between the advection dominated pumping zone and the underlying porous medium. This flux is associated to permanent mass retention in the bed which implies that a fraction of the solute entering the bed has infinite residence time. When the dispersivities are high, the purely advective model can significantly underestimate the mass transfer into the bed at late times. In the dispersion dominated region, where the RTD diverges from the prediction of the advective model, the $R$ function exhibits a pattern similar to a power law. If the dispersion tensor was constant, $R(t)$ would diverge from the pure advective case following a power law with exponent $-1 / 2\left(R \sim t^{-1 / 2}\right)$. Since the dispersion tensor is related to the seepage velocity according to (18), thus varying with depth and assuming a constant value where the underflow is not affected by pumping, the tail of the $R$ function shows a more complex behavior resulting in $R \sim t^{-1 / 2}$ only at later time scales (Figure 6). At earlier times, pore-scale dispersion induces a pattern that can be approximated by a power law with exponents lower than $-1 / 2$. As shown in Figure 7, the seepage velocity $u_{s}^{*}$ affects the tail of $R$ in the dispersion dominated region inducing longer residence times as $u_{s}^{*}$ increases, consistently with the increase of the components of the dispersion tensor (equation (18)). Physically, mass is dislocated by dispersion into deeper streamlines, thus making the solute path in the bed longer. Nevertheless, for the range of values determined in laboratory experiments by Elliott and Brooks [1997a], namely, $u_{s}^{*}=0.04-0.11$, and indicated as typical of field situations where the form drag dominates the total drag, the variations of $R$ due to dispersion for a given $U_{u f}^{*}$ are rather small.

[34] Depending on the values of the underflow velocity and of the dispersivities, the effect of dispersion is visible at different time scales. The tails of the $R$ function can be divided in different time ranges to which different functional forms apply. To illustrate this concept, we consider the case of $u_{s}^{*}=0.1$ presented in Figure 6. For $U_{u f}^{*} \approx 0$ the late time behavior of the residence time function is characterized by a first power law region where $R \sim t^{-1}$ and a final region, dominated by dispersion, where $R \sim t^{-1 / 2}$. This is the limit case characterized by the highest mass transfer. It should be noted that situations where $U_{u f}^{*} \approx 0$ can be found when the speed of bed form propagation is approximately equal to the groundwater velocity induced by the stream gradient. For $U_{u f}^{*}$ sufficiently higher than zero but $U_{u f}^{*}<0.4\left(t_{c}^{*}>10\right)$ and relatively small dispersivities $\left(\alpha_{L}^{*} \ll 0.6\right)$, the tail of residence time function shows a power law pattern with $R \sim t^{-1}$ in the time range where the advective pumping process is dominant, a second range where the function decays exponentially $\left(R \sim e^{-t / t_{d}}\right)$ due to the limiting effect of the underflow, and then another temporal region, when the exchange process is dominated by mechanical dispersion, where $R$ tends to be asymptotically proportional to $t^{-1 / 2}$ but with slightly lower exponents at earlier times. For $0.4<$ $U_{u f}^{*}<1$, the tail is characterized by an exponential region followed by a power law region where $R \sim t^{-1 / 2}$ at late time.

[35] When $U_{u f}^{*} \gg 1$ the effect of advective pumping early becomes negligible compared to the effect of dispersion which is the only process responsible for the long-term behavior of the residence time function. The result would not sensibly change if the effect of turnover is accounted for. Indeed, pore-scale dispersion can well explain the discrepancy observed by Elliott and Brooks [1997b] between the prediction of their turnover model and their experimental 
results, as discussed by Packman and Brooks [2001]: The turnover model greatly underestimated the exchange for moving bed forms, especially in case of high propagation speeds, and the penetrated mass was found to be increasing with $t^{1 / 2}$

[36] As the dispersivity of the porous medium increases, the exchange process is dominated by dispersion at
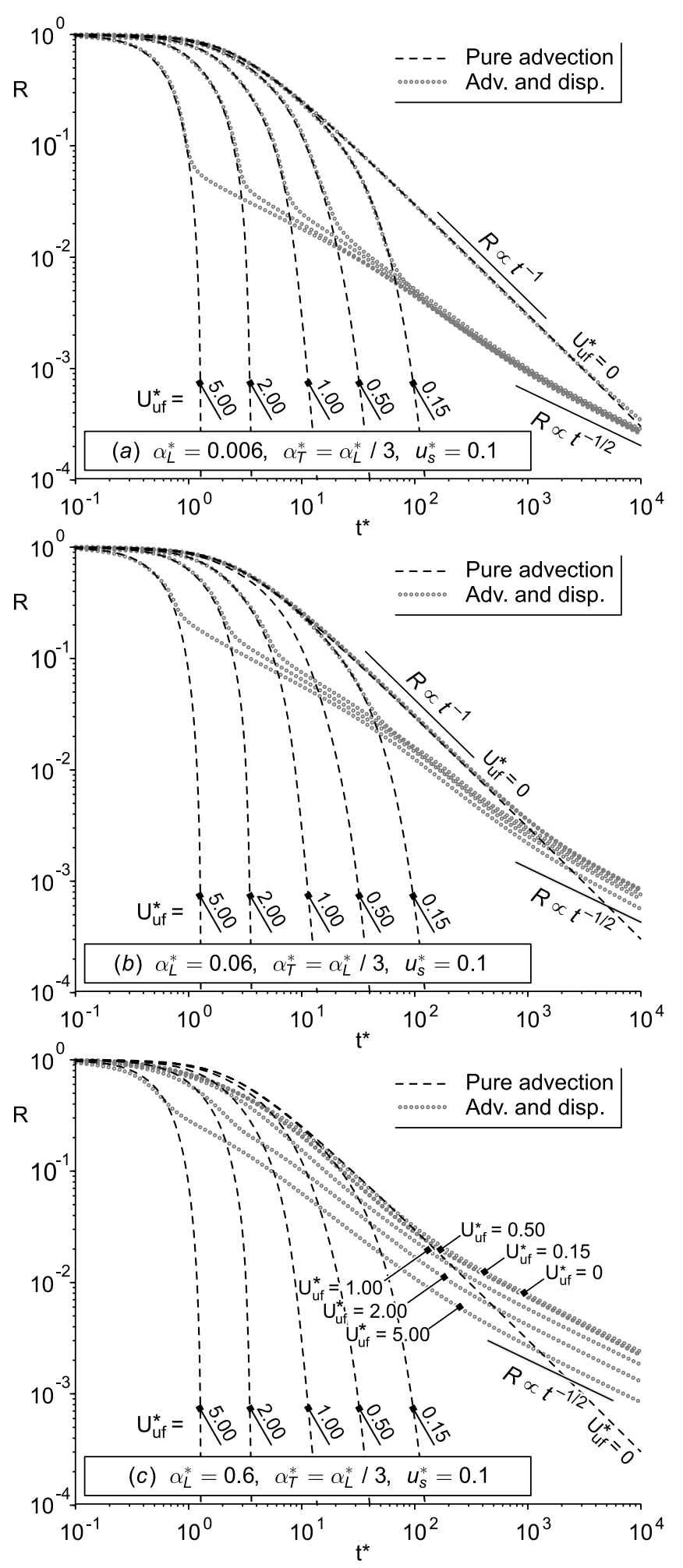

increasingly earlier time scales. When the dispersivity is high, as shown in Figure 6c for $\alpha^{*}{ }_{L}=0.6$, dispersion is the dominant process affecting the whole tail of $R$. In this situation the effect of the vertical variability of the dispersion tensor is even more evident: The slope of $R$ in a log-log graph is lower than $-1 / 2$ in a wide temporal range and $R \sim$ $t^{-1 / 2}$ only at late times.

\section{Discussion}

[37] In experimental tracer tests, the late time behavior of breakthrough curves is recognized to be the signature of long-term retention typically caused by transient storage. The results of this work show that, as far as bed forminduced hyporheic exchange is concerned, distinct functional forms of the residence time distributions apply to advection and dispersion-dominated flows in appropriate time ranges. The temporal extent of these regions depends on the characteristics of the surface flow and the sediment bed. Although these results were derived for a semi-infinite bed, the description of the results can be generalized to the case of a permeable bed of limited thickness.

[38] In the case of bed form-induced exchange, when penetration is limited by an impermeable boundary placed deeper underneath the pumping-dominated advective zone, the final part of the residence time distribution displays an exponential decay. This behavior characterizes the temporal range in which the limiting effect of the impermeable boundary significantly affects the vertical concentration profile, whereas at earlier times the distributions remain unaltered. On the other hand, if the thickness of the permeable layer is small enough to affect the flow paths induced by the bed form-associated pumping effect, and if the relative underflow velocity is low enough to allow for the formation of stagnation points in the flow field within the bed, then the maximum residence time is infinite (at least from a mathematical perspective) but the associated probability must rapidly decrease to zero at late times, most probably exponentially.

[39] In the first modeling approach, the TSM [Bencala and Walters, 1983], the residence time distribution was implicitly assumed exponential. A first advancement was proposed by Choi et al. [2000] who noticed that the superposition of more than one storage process could be modeled by the additions of other exponential RTDs. A further development was then proposed by Wörman et al. [2002] who suggested that the PDF of the residence time of bed form-induced hyporheic exchange could be approximated by a lognormal distribution. For stationary bed forms

Figure 6. Residence time functions of hyporheic exchange in case of advective-dispersive transport in the porous medium for different values of the dispersivity $\alpha_{L}^{*}$ and relative underflow velocity $U_{u f}^{*}$. In the simulations the ratio $\alpha_{T}^{*} / a_{L}^{*}$ and the velocity $u_{s}^{*}$ are kept constant and equal to $1 / 3$ and 0.1 , respectively. Pore-scale dispersion induces longer tails in the $R$ function which tends asymptotically to follow a power law scaling as $R \sim t^{-1 / 2}$. At earlier times in the temporal region dominated by dispersion, the slope of the function in a log-log graph is not constant and can assume values lower than $-1 / 2$ as consequence of the vertical variability of the dispersion tensor. 


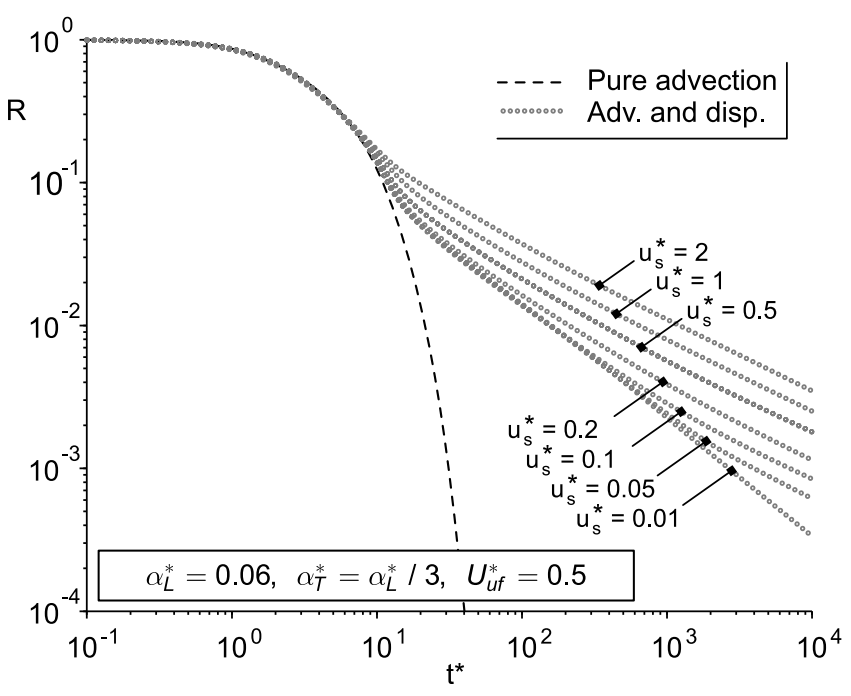

Figure 7. Effect of the seepage velocity induced by the stream gradient, $u_{s}^{*}$, on the residence time function, $R$, for a given relative underflow velocity, $U_{u f}^{*}$. The curves are generated by keeping $U_{u f}^{*}$ constant, which implies that an increase of $u_{s}^{*}$ is balanced by an increase of the bed form propagation celerity, $U_{b}^{*}$. Higher velocities $u_{s}^{*}$ produce higher dispersion coefficients. which in turn increase the probability associated to longer residence times.

and typical groundwater velocities induced by the stream gradient, a comparison in linear scale shows that, for properly chosen parameters, a lognormal distribution can be a good approximation of (23). Nevertheless, when the distributions are compared in a logarithmic scale, their tail behavior appears substantially different, and even more different if the effect of dispersion is accounted for. The importance of comparing model simulations to concentration data in $\log$ (concentration) scale at late time has been increasingly recognized in the last few years. Recent studies have shown that tracer breakthrough curves often exhibit a long tail behavior that may be in the form of power law patterns. At late times, BTCs are proportional to the corresponding PDF of the residence time, and hence to the derivative of the function R. Haggerty and Wondzell [2002] reported data from a tracer test in a second-order mountain stream in which the tail of the breakthrough curve followed a power law scaling as $t^{-1.3}\left(R \sim t^{-0.3}\right)$ over at least 1.5 orders of magnitude in time. Gooseff et al. [2003] performed tracer tests in different reaches of a mountain stream and found that the experimental curves could be well approximated using a truncated power law RTD to represent transient storage, with exponents ranging from -1.6 to $-1.3\left(R \sim t^{-0.6}\right.$ to $\left.t^{-0.3}\right)$. More recently Gooseff et al. [2007] reported data from tracer tests performed in streams of different channel complexity. Part of them could be represented by a conventional model assuming an exponential RTD, but a few of them displayed power law patterns with exponents in the range between -1.9 and $-1.7\left(R \sim t^{-0.9}\right.$ to $\left.R \sim t^{-0.7}\right)$. Although the substrate material of the streambed in these tracer studies was primarily gravel, cobbles and bedrock, to which the pumping theory does not apply, it can be noticed that the exponents of the power law reported lie chiefly between -2 and $-3 / 2$, the values associated to advective pumping and dispersion, respectively. Power law tail behavior with exponents in the range between -1.9 and -1.7 ( $R \sim t^{-0.9}$ to $R \sim t^{-0.7}$ ) has recently been confirmed also through numerical simulations of advective-dispersive flows using a commercial code (COMSOL) [Cardenas et al., 2008]. Intermediate exponents between -2 and $-3 / 2$ have been shown to arise as a consequence of the vertical variability of the dispersion tensor, but can also be due to the multiconvolution of the residence time distributions of many bed forms arranged in sequence. Heterogeneity of the porous medium may also play a role in defining the apparent exponent of the power law [Sawyer and Cardenas, 2009]. On the other hand, exponents higher than $-3 / 2\left(R \sim t^{-1 / 2}\right)$, indicating a longer retention of solutes in the bed, cannot be explained within the modeling framework presented here. Other types of stream-aquifer interactions besides bed form-driven exchange may concur to determine power law breakthrough curves in stream tracer studies.

[40] The results of this paper show that the hyporheic exchange is a complex combination of advective and dispersive transport processes. Real conditions present a even higher complexity due to a wide range of topography and heterogeneity. Such complexity makes it very difficult to measure the processes in the field with adequate detail. Tracer tests remain the simplest way to extract information about hyporheic exchange in field applications. The uncertainty arising from the superposition of surface and subsurface retention processes requires that the number of parameters involved in transport models is kept to the minimum necessary to get an acceptable representation of the observed breakthrough curves.

[41] The introduction of two distinct exponential distributions, as suggested by some authors [Harvey et al., 2005; Briggs et al., 2009], is the simplest way to move from a single RTD, as for instance in the transient storage model [Bencala and Walters, 1983], to multiple RTDs. Recent contributions by Briggs et al. [2009] and recent observations by the authors (work in progress) show that models with two exponential RTDs are flexible enough to be successfully calibrated with field tracer data. The multiple exponential model definitely improves the fitting of the BTCs over a larger time range. However, this approach may turn out to be only a mere introduction of additional calibration parameters. While exponential distributions are a suitable model for retention in storage domains of finite volume (e.g., surface dead zones, hyporheic zones of limited thickness [Zaramella et al., 2003]), it is clear that they are not always a physically based model of advective-dispersive hyporheic transport processes. When advective pumping or mechanical dispersion in the porous medium are dominant processes, the use of two exponential RTDs may still provide acceptable fitting of tracer BTCs. This may happen for three distinct reasons. First, there is a chance that the system is made of a combination of finite volume hyporheic zones in parallel, a special case where the two exponential RTD model is indeed physically sound [Choi et al., 2000]. Second, the model has a number of calibration parameters large enough to compensate for the lack of physical correspondence. Third, the time scales of observations are relatively short due to constraints imposed by instrumental detection limits and they capture only a very limited range of time scales, much less than the actual distribution of hyporheic 
exchange times. Power law patterns require accurate measures over a wide range of time scales to become clearly recognizable. Over smaller temporal ranges the difference between a power law and an exponential behavior may not be detectable without uncertainty. However, when the tails of the observed breakthrough curves exhibit seemingly power law patterns with exponents close to $-2\left(R \sim t^{-1}\right)$ or $-3 / 2(R \sim$ $\left.t^{-1 / 2}\right)$, this can be regarded respectively as a fingerprint of bed form-induced advective pumping or subsurface dispersion affecting a significant portion of the porous medium.

\section{Conclusions}

[42] Simulations have shown that the residence time distributions of bed form-induced hyporheic exchange are characterized by a complex tail behavior due to the combination of advective transport and pore-scale dispersion in the porous medium. At different stages of the exchange process, the RTDs are characterized by different functional forms. When the bed form-induced advective pumping is a dominant process and the flow paths are not limited by an underlying groundwater flow or by an impermeable boundary, the probability density function of the residence time, $r(t)$, asymptotically tends to a power law decaying as $t^{-2}\left(R \sim t^{-1}\right)$. The combination of the seepage velocity induced by the stream gradient, and the celerity of the translating bed forms in case of a moving bed, results in a limitation of the advective transport into deeper portions of the porous medium and causes the PDF to rapidly decay to zero if only advection is accounted for. When the underflow velocity relative to a frame of reference moving at the speed of bed form propagation is lower than the maximum pumping-induced velocity, $r(t)$ decays exponentially, $r \sim$ $e^{-t / t_{d}}\left(R \sim e^{-t / t_{d}}\right)$, and the slope in a semi-log graph depends on a properly defined time scale $t_{d}$. When the relative velocity of the underflow is higher than the maximum pumping-induced velocity, the residence time varies in a bounded time interval. Pore-scale dispersion counteracts the limiting effect of the relative underflow velocity producing longer tails in the RTDs. In the time range dominated by dispersion the PDF $r(t)$ follows a power law tending to $r \sim$ $t^{-3 / 2}$ at late times $\left(R \sim t^{-1 / 2}\right)$, and slightly lower exponents can be found at earlier times as a consequence of the vertical variability of the dispersion tensor.

[43] The results presented highlight that both exponential and power law patterns in the late time behavior of solute breakthrough curves can be associated to hyporheic exchange. When the first prevails, the thickness of the hyporheic zone is limited, whereas, when the second prevails, there is deeper exchange with the bed that may be due to advective pumping or mechanical dispersion. The identification of power law patterns in the breakthrough curves of tracer tests requires measurement over a wide range of time scales, often not available because of instrumental detection limits. This limitation can make calibration models assuming an exponential RTD to represent hyporheic retention sufficient to well reproduce the observed breakthrough curves, even when the mass transfer is not limited to a surficial layer of the bed. However, power laws with exponents in the range from -2 to $-3 / 2$, when visible, should be regarded as a particular signature of hyporheic exchange affecting a significant thickness of the streambed.

\section{References}

Bencala, K. E., and R. A. Walters (1983), Simulation of solute transport in a mountain pool-and-riffle stream: A transient storage model, Water Resour. Res., 19(3), 718-724, doi:10.1029/WR019i003p00718.

Benekos, D. I. (2005), On the determination of transverse dispersivity: Experiments and simulations in a helix and a cochlea, Ph.D. dissertation, Dep. of Civ. and Environ. Eng., Stanford Univ., Stanford, Calif.

Boano, F., C. Camporeale, R. Revelli, and L. Ridolfi (2006a), Sinuositydriven hyporheic exchange in meandering rivers, Geophys. Res. Lett., 33, L18406, doi:10.1029/2006GL027630.

Boano, F., R. Revelli, and L. Ridolfi (2006b), Bedform-induced hyporheic exchange with unsteady flow, Adv. Water Resour., 30(1), 148-156, doi:10.1016/j.advwatres.2006.03.004.

Boano, F., A. I. Packman, A. Cortis, R. Revelli, and L. Ridolfi (2007), A continuous time random walk approach to the stream transport of solutes, Water Resour. Res., 43, W10425, doi:10.1029/2007WR006062.

Boano, F., R. Revelli, and L. Ridolfi (2008), Reduction of the hyporheic zone volume due to the stream-aquifer interaction, Geophys. Res. Lett., 35, L09401, doi:10.1029/2008GL033554.

Briggs, M. A., M. N. Gooseff, C. D. Arp, and M. A. Baker (2009), A method for estimating surface transient storage parameters for streams with concurrent hyporheic storage, Water Resour. Res., 45, W00D27, doi:10.1029/2008WR006959.

Brinkman, H. C. (1949), A calculation of the viscous force exerted by a flowing fluid on a dense swarm of particles, Appl. Sci. Res., 1(1), 27-34, doi:10.1007/BF02120313.

Calhoun, D., and R. J. LeVeque (2000), A Cartesian grid finite-volume method for the advection-diffusion equation in irregular geometries, J. Comput. Phys., 157(1), 143-180, doi:10.1006/jcph.1999.6369.

Cardenas, M. B. (2003), Three-dimensional model of modern channel bend deposits, Water Resour. Res., 39(6), 1141, doi:10.1029/2002WR001383.

Cardenas, M. B. (2008), The effect of river bend morphology on flow and timescales of surface water-groundwater exchange across pointbars, J. Hydrol., 362(1-2), 134-141, doi:10.1016/j.jhydrol.2008.08.018.

Cardenas, M. B., and J. L. Wilson (2007a), Dunes, turbulent eddies, and interfacial exchange with permeable sediments, Water Resour. Res., 43, W08412, doi:10.1029/2006WR005787.

Cardenas, M. B., and J. L. Wilson (2007b), Exchange across a sedimentwater interface with ambient groundwater discharge, J. Hydrol., 346(3-4), 69-80, doi:10.1016/j.jhydrol.2007.08.019.

Cardenas, M. B., J. L. Wilson, and R. Haggerty (2008), Residence times of bedform-driven hyporheic exchange, Adv. Water Resour., 31(10), 13821386, doi:10.1016/j.advwatres.2008.07.006.

Choi, J., J. W. Harvey, and M. H. Conklin (2000), Characterizing multiple timescales of stream and storage zone interaction that affect solute fate and transport in streams, Water Resour. Res., 36(6), 1511-1518, doi:10.1029/2000WR900051.

Elliott, A. H., and N. H. Brooks (1997a), Transfer of nonsorbing solutes to a streambed with bed forms: Theory, Water Resour. Res., 33(1), 123-136, doi:10.1029/96WR02784.

Elliott, A. H., and N. H. Brooks (1997b), Transfer of nonsorbing solutes to a streambed with bed forms: Laboratory experiments, Water Resour. Res., 33(1), 137-151, doi:10.1029/96WR02783.

Fehlman, H. M. (1985), Resistance components and velocity distribution of open channel flows over bed form, M.S. thesis, Colo. State Univ., Fort Collins.

Fischer, H. B., J. E. List, C. R. Koh, J. Imberger, and N. H. Brooks (1979), Mixing in Inland and Coastal Waters, Academic, San Diego, Calif.

Gibert, J., D. Danielopol, and J. A. Stanford (1994), Groundwater Ecology, Academic, San Diego, Calif.

Gooseff, M. N., S. M. Wondzell, R. Haggerty, and J. Anderson (2003), Comparing transient storage modeling and residence time distribution (RTD) analysis in geomorphically varied reaches in the Lookout Creek basin, Oregon, USA, Adv. Water Resour., 26(9), 925-937, doi:10.1016/S0309-1708(03)00105-2.

Gooseff, M. N., R. O. Hall Jr., and J. L. Tank (2007), Relating transient storage to channel complexity in streams of varying land use in Jackson Hole, Wyoming, Water Resour. Res., 43, W01417, doi:10.1029/ 2005WR004626.

Haggerty, R., and S. M. Wondzell (2002), Power-law residence time distribution in the hyporheic zone of a 2nd-order mountain stream, Geophys. Res. Lett., 29(13), 1640, doi:10.1029/2002GL014743.

Haggerty, R., S. A. McKenna, and L. C. Meigs (2000), On the late-time behavior of tracer test breakthrough curves, Water Resour. Res., 36(12), 3467-3479, doi:10.1029/2000WR900214. 
Harvey, J. W., and K. E. Bencala (1993), The effect of streambed topography on surface-subsurface water exchange in mountain catchments, Water Resour. Res., 29(1), 89-98, doi:10.1029/92WR01960.

Harvey, J. W., J. E. Saiers, and J. T. Newlin (2005), Solute transport and storage mechanisms in wetlands of the Everglades, south Florida, Water Resour. Res., 41, W05009, doi:10.1029/2004WR003507.

Jones, J. B., and P. J. Mulholland (2000), Streams and Ground Waters, Academic, San Diego, Calif.

LeVeque, R. J. (1997), Wave propagation algorithms for multidimensional hyperbolic systems, J. Comput. Phys., 131(2), 327-353, doi:10.1006/ jcph.1996.5603.

Marion, A., and M. Zaramella (2005), A residence time model for streamsubsurface exchange of contaminants, Acta Geophys. Polonica, 53(4), 527.

Marion, A., and M. Zaramella (2006), Effects of velocity gradients and secondary flow on the dispersion of solutes in a meandering channel, J. Hydraul. Eng., 132(12), 1295-1302, doi:10.1061/(ASCE)07339429(2006)132:12(1295).

Marion, A., M. Bellinello, I. Guymer, and A. I. Packman (2002), Effect of bed form geometry on the penetration of nonreactive solutes into a streambed, Water Resour. Res., 38(10), 1209, doi:10.1029/2001WR000264.

Marion, A., M. Zaramella, and A. I. Packman (2003), Parameter estimation of the transient storage model for stream-subsurface exchange, J. Environ. Eng., 129(5), 456-463, doi:10.1061/(ASCE)0733-9372(2003) 129:5(456).

Marion, A., A. I. Packman, M. Zaramella, and A. Bottacin-Busolin (2008a), Hyporheic flows in stratified beds, Water Resour. Res., 44, W09433, doi:10.1029/2007WR006079.

Marion, A., M. Zaramella, and A. Bottacin-Busolin (2008b), Solute transport in rivers with multiple storage zones: The STIR model, Water Resour Res., 44, W10406, doi:10.1029/2008WR007037.

Nagaoka, H., and A. J. Ohgaki (1990), Mass transfer mechanism in a porous riverbed, Water Res., 24(4), 417-425, doi:10.1016/0043-1354 (90)90223-S

Packman, A. I., and N. H. Brooks (2001), Hyporheic exchange of solutes and colloids with moving bed forms, Water Resour. Res., 37(10), 2591-2605, doi:10.1029/2001WR000477.

Packman, A. I., N. H. Brooks, and J. J. Morgan (2000a), A physicochemical model for colloid exchange between a stream and a sand streambed with bed forms, Water Resour. Res., 36(8), 2351-2361, doi:10.1029/ 2000WR900059.

Packman, A. I., N. H. Brooks, and J. J. Morgan (2000b), Kaolinite exchange between a stream and streambed: Laboratory experiments and validation of a colloid transport model, Water Resour. Res., 36(8), 2363-2372, doi:10.1029/2000WR900058.

Qian, Q., V. R. Voller, and H. G. Stefan (2008), A vertical dispersion model for solute exchange induced by underflow and periodic hyporheic flow in a stream gravel bed, Water Resour. Res., 44, W07422, doi:10.1029/ 2007WR006366.

Runkel, R. L. (1998), One-dimensional transport with inflow and storage (OTIS): A solute transport model for streams and rivers, U.S. Geol. Surv. Water Resour. Invest. Rep., 98-4018, 73 pp.

Salehin, M., A. I. Packman, and M. Paradis (2004), Hyporheic exchange with heterogeneous streambeds: Laboratory experiments and modeling, Water Resour. Res., 40, W11504, doi:10.1029/2003WR002567.

Sawyer, A. H., and M. B. Cardenas (2009), Hyporheic flow and residence time distributions in heterogeneous cross-bedded sediment, Water Resour. Res., 45, W08406, doi:10.1029/2008WR007632.

Shimizu, Y., T. Tsujimoto, and H. Nakagawa (1990), Experiment and microscopic modeling of flow in highly permeable porous medium under free-surface flow, J. Hydrosci. Hydraul. Eng., 8(1), 69-78.

Tonina, D., and J. M. Buffington (2007), Hyporheic exchange in gravel bed rivers with pool-riffle morphology: Laboratory experiments and threedimensional modeling, Water Resour. Res., 43, W01421, doi:10.1029/ 2005WR004328.

Vittal, N., K. J. Ranga Raju, and R. J. Garde (1977), Resistance of twodimensional triangular roughness, J. Hydraul. Res., 15(1), 19-36.

Wörman, A., A. I. Packman, H. Johansson, and K. Jonsson (2002), Effect of flow-induced exchange in hyporheic zones on longitudinal transport of solutes in streams and rivers, Water Resour. Res., 38(1), 1001, doi:10.1029/2001WR000769.

Wörman, A., A. I. Packman, L. Marklund, J. W. Harvey, and S. H. Stone (2006), Exact three-dimensional spectral solution to surface-groundwater interactions with arbitrary surface topography, Geophys. Res. Lett., 33, L07402, doi:10.1029/2006GL025747.

Wörman, A., A. I. Packman, L. Marklund, J. W. Harvey, and S. H. Stone (2007), Fractal topography and subsurface water flows from fluvial bedforms to the continental shield, Geophys. Res. Lett., 34, L07402, doi:10.1029/2007GL029426.

Zaramella, M., A. I. Packman, and A. Marion (2003), Application of the transient storage model to analyze advective hyporheic exchange with deep and shallow sediment beds, Water Resour. Res., 39(7), 1198, doi:10.1029/2002WR001344.

Zheng, C., and G. D. Bennett (1995), Applied Contaminant Transport Modeling, John Wiley, Hoboken, N. J.

Zhou, D., and C. Mendoza (1993), Flow through porous bed of turbulent stream, J. Eng. Mech., 119(2), 365-383, doi:10.1061/(ASCE)07339399(1993)119:2(365).

A. Bottacin-Busolin and A. Marion, Department of Hydraulic, Maritime, Environmental and Geotechnical Engineering, University of Padova, Via Loredan 20, Padua I-35131, Italy. (andrea.bottacin@unipd.it) 